\title{
Deep germanium etching using time multiplexed plasma etching
}

Maxime Darnon, Mathieu de Lafontaine, Maïté Volatier, Simon Fafard, Richard Arès, Abdelatif Jaouad, and Vincent Aimez

Citation: Journal of Vacuum Science \& Technology B, Nanotechnology and Microelectronics: Materials, Processing, Measurement, and Phenomena 33, 060605 (2015);

View online: https://doi.org/10.1116/1.4936112

View Table of Contents: http://avs.scitation.org/toc/jvb/33/6

Published by the American Vacuum Society

\section{Articles you may be interested in}

Selective dry etching of silicon with respect to germanium

Applied Physics Letters 56, 1436 (1998); 10.1063/1.102490

Plasma etching applications in concentrated photovoltaic cell fabrication

AIP Conference Proceedings 1766, 060001 (2016); 10.1063/1.4962091

Precision plasma etching of $\mathrm{Si}, \mathrm{Ge}$, and $\mathrm{Ge}: \mathrm{P}$ by $\mathrm{SF}_{6}$ with added $\mathrm{O}_{2}$

Journal of Vacuum Science \& Technology A: Vacuum, Surfaces, and Films 32, 031302 (2014);

10.1116/1.4868615

Chemical downstream etching of $\mathrm{Ge}, \mathrm{Si}$, and $\mathrm{SiN}_{\mathrm{X}}$ films

Journal of Vacuum Science \& Technology B, Nanotechnology and Microelectronics: Materials, Processing, Measurement, and Phenomena 34, 052003 (2016); 10.1116/1.4961944

Quasi-atomic layer etching of silicon nitride

Journal of Vacuum Science \& Technology A: Vacuum, Surfaces, and Films 35, 01 A102 (2016);

$10.1116 / 1.4967236$

Germanium etching in high density plasmas for $0.18 \mu \mathrm{m}$ complementary metal-oxide-semiconductor gate patterning applications

Journal of Vacuum Science \& Technology B: Microelectronics and Nanometer Structures Processing,

Measurement, and Phenomena 16, 1833 (1998); 10.1116/1.590094

Contact Hiden Analytical for further details: W www.HidenAnalytical.com E info@hiden.co.uk CLICK TO VIEW our product catalogue

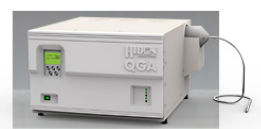

Gas Analysis

dynamic measurement of reaction gas streams catalysis and thermal analysis molecular beam studies dissolved species probes , dissolved spectisprobes

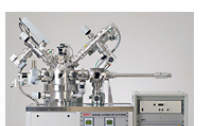

Surface Science

UHVTPD

end point detection in ion beam etch elemental imaging - surface mapping

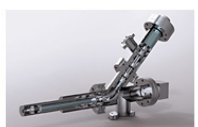

Plasma Diagnostics

plasma source characterization etch and deposition process reaction kinetic studies analysis of neutral and radical species

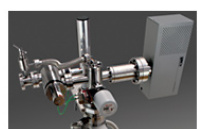

Vacuum Analysis partial pressure measurement and control of process gases reactive sputter process control vacuum diagnostics vacuum coating process monitoring 


\title{
Deep germanium etching using time multiplexed plasma etching
}

\author{
Maxime Darnon, ${ }^{\text {a) }}$ Mathieu de Lafontaine, Maïté Volatier, Simon Fafard, Richard Arès, \\ Abdelatif Jaouad, and Vincent Aimez \\ Laboratoire Nanotechnologies Nanosystèmes (LN2) - CNRS UMI-3463, Institut Interdisciplinaire \\ d'Innovation Technologique (3IT), Université de Sherbrooke, 3000 Boulevard de l'Université, Sherbrooke, \\ JIK OA5 Québec, Canada
}

(Received 25 August 2015; accepted 30 October 2015; published 20 November 2015)

\begin{abstract}
There is a growing need for patterning germanium for photonic and photovoltaics applications. In this paper, the authors use a time multiplexed plasma etch process (Bosch process) to deep etch a germanium substrate. They show that germanium etching presents a strong aspect ratio dependent etching and that patterns present scallops mostly on the upper part (aspect ratio below 0.8 ). Passivation layers are formed during the passivation step by neutrals' deposition and are reinforced during the etching step by the redeposition of sputtered fluorocarbon species from the etch front. When the sidewalls are passivated, reactive neutrals diffuse through Knudsen-like diffusion down to the bottom of the pattern to etch the germanium. The Knudsen-like diffusion is responsible for the aspect ratio dependent etching and makes difficult the etching of holes with aspect ratios above 10 while trenches with aspect ratio of 17 are still etched faster than $2 \mu \mathrm{m} / \mathrm{min}$. @ 2015 American Vacuum Society. [http://dx.doi.org/10.1116/1.4936112]
\end{abstract}

\section{INTRODUCTION}

Germanium is a semiconductor that has been used for years for many applications including microelectronics, optoelectronics, and photovoltaics. There has been a regain of interest in germanium patterning recently, especially for photonic integrated circuits. In addition, germanium is the common substrate to III-V materials' growth, thanks to its lattice match with GaAs enabling epitaxy of III-V materials on germanium for photonic and photovoltaic applications. The high aspect ratio germanium patterns have been formed using growth, ${ }^{1}$ electrochemical etching, ${ }^{2}$ and plasma etching, ${ }^{3}$ the latter one being the most appropriate technique for local and defined patterns' formation.

Plasma etching of germanium has been investigated by many papers that reported the capability of germanium etching in chlorine- and fluorine-based plasma processes. ${ }^{4-12}$ Using chlorine-based plasmas, the etch rate is generally below $1 \mu \mathrm{m} / \mathrm{min}$ (except for one report at $4 \mu \mathrm{m} / \mathrm{min}$ by Choe et al. ${ }^{9}$ ) with isotropic etching unless passivating species such as GeN (Ref. 8) are formed during the process. A cyclic process with oxidation by air exposure was also reported as a solution for anisotropic etching in chlorine-based plasma. ${ }^{3} \mathrm{~A}$ much faster etch rate is reported in fluorine plasma with isotropic behavior. ${ }^{4-6,10-12}$ The etch mechanisms were reported to be quite similar to the ones of silicon, except for the inability for $\mathrm{GeO}_{\mathrm{x}} \mathrm{F}_{\mathrm{y}}$ to inhibit the etching at room temperature (compared to $\mathrm{SiO}_{\mathrm{x}} \mathrm{F}_{\mathrm{y}}$ that quenches the etching) ${ }^{5,6}$ and the different sulfides stoichiometry $\left(\mathrm{GeS}_{2}\right.$ compared to $\left.\mathrm{SiS}\right)$ and volatility $\left(\mathrm{GeS}_{2}\right.$ is less volatile than $\left.\mathrm{SiS}\right){ }^{4,13}$ In general, germanium etching is faster than silicon etching in fluorinebased plasmas. ${ }^{4}$ Some anisotropic etching of germanium in fluorine based plasma has been reported for $\mathrm{SF}_{6} / \mathrm{O}_{2}$ plasma with a large concentration of oxygen ${ }^{12}$ and in $\mathrm{a} \mathrm{CF}_{4}$-based plasma in a silicon rich environment. ${ }^{11}$

${ }^{a)}$ Electronic mail: maxime.darnon@usherbrooke.ca
Deep silicon etching is generally performed using time multiplexed plasma etching, also-called the Bosch process. ${ }^{14}$ It consists in the switching between fast and isotropic etching and passivation deposition steps. ${ }^{15}$ Such processes provide high selectivity to resist or silicon oxide mask, fast etch rate $(>1 \mu \mathrm{m} / \mathrm{min})$, and anisotropic profiles at the expense of a sidewall roughness coming from the alternating behavior of the process. This roughness has a scallop shape, each scallop being formed by an individual etch step, and is commonly referred to as scalloping.

Despite similarities between the silicon and germanium etching in fluorine-based plasmas, there is no report in the literature of patterns formation using deep germanium etching with a Bosch process. Williams et al. reported the capability of germanium etching using a Bosch process, ${ }^{16}$ and Schicho et al. reported the formation of black germanium using a Bosch process. ${ }^{17}$ However, none of those papers discuss the pattern formation with such a process.

In this letter, we report deep germanium etching using a Bosch process developed for silicon etching. We discuss the evolution of the etched depth and profile along the process and precise the mechanisms of the passivation and the etching steps.

\section{EXPERIMENT}

The experiments are performed using $\sim 170 \mu \mathrm{m}$-thick germanium wafers (100) with $6^{\circ}$ miscut crystalline orientation from (100) as typically dedicated to III-V growth. The wafers are coated with $1.5 \mu \mathrm{m}$ of plasma enhanced chemical vapor deposition $\mathrm{SiO}_{2}$ acting as a hard mask. The hard mask is patterned with holes and trenches of diameter/width comprised between 5 and $50 \mu \mathrm{m}$ defined by photolithography and plasma etching $\left(\mathrm{CF}_{4} / \mathrm{C}_{4} \mathrm{~F}_{8}\right.$ etch process). The photoresist is removed before germanium etching.

Germanium etching is performed in a SPTS advanced silicon etch featuring inductively coupled plasma. The chamber operates in a time multiplexed mode (Bosch process). The 

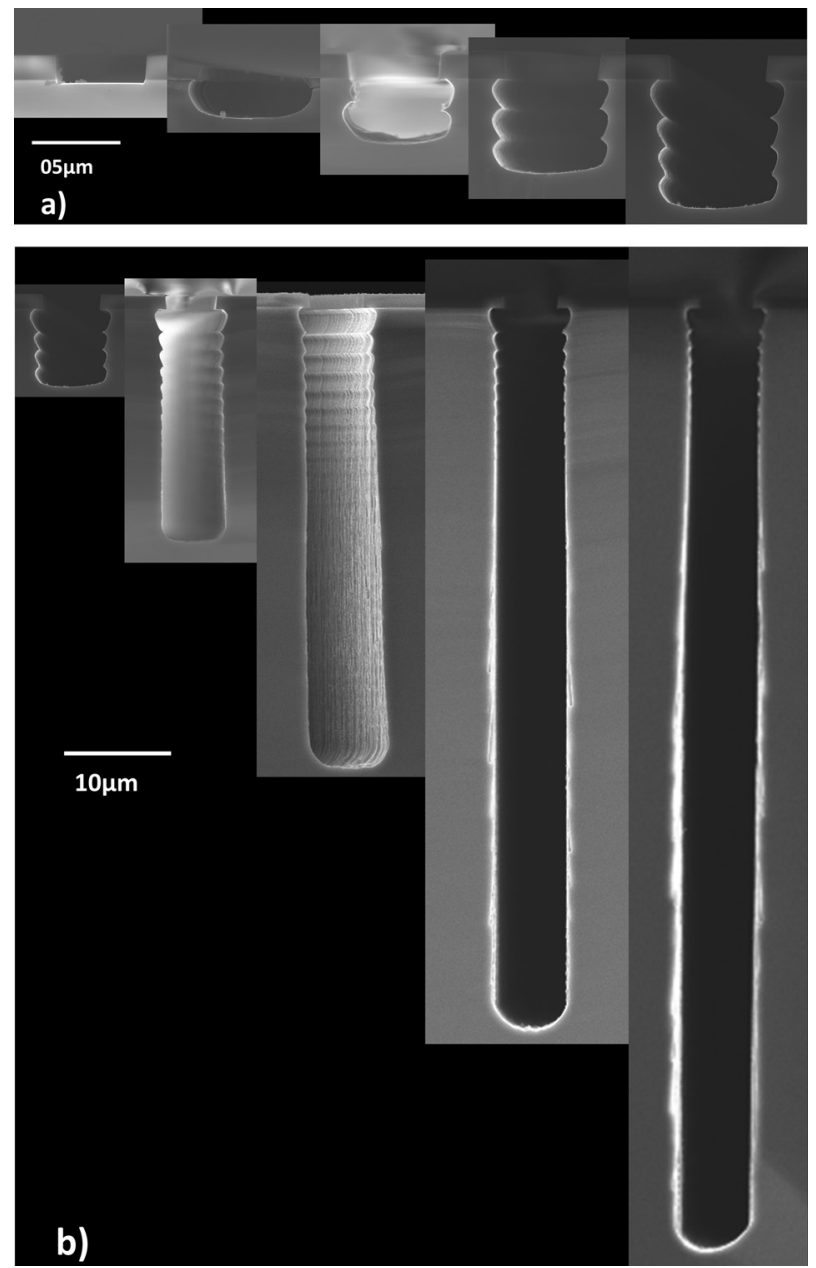

FIG. 1. Cross section images of $5 \mu$ m-wide trenches etched in germanium with from left to right (a) 0, 1, 2, 3, 4, (b) 4, 15, 30, 60 and 90 cycles.

inductive source power operates at $13.56 \mathrm{MHz}$. An additional power supply at $13.56 \mathrm{MHz}$ is used to bias the sample holder. The wafers are clamped with electrostatic clamping on the cathode that is thermally regulated at $20^{\circ} \mathrm{C}$. Helium flux ensures thermal conduction between the cathode and the wafer. The details on the plasma chamber can be found in the literature. ${ }^{18}$ Pieces of the germanium wafer are patched on a 4 in. quartz wafer with crystal bound ${ }^{\mathrm{TM}}$ to maintain the sample and ensure thermalization between the germanium and the quartz wafer. The process used consists in $7 \mathrm{~s}$ of passivation $\left(\mathrm{C}_{4} \mathrm{~F}_{8}\right.$ plasma without bias power) and $13 \mathrm{~s}$ of etching $\left(\mathrm{SF}_{6} / \mathrm{O}_{2}\right.$ with $9 \% \mathrm{O}_{2}$ plasma with $18 \mathrm{~W}$ of bias power). This process was optimized for silicon etching. Before each process, the chamber was cleaned with a long $\mathrm{O}_{2}$ plasma and seasoned by processing a bare quartz wafer for more than $5 \mathrm{~min}$.

The pattern profiles are observed using a Supra $55 \mathrm{VP}$ scanning electron microscope (SEM) from Zeiss.

\section{RESULTS}

\section{A. Observation of germanium etching using the Bosch process}

We first analyzed the evolution of the etch profile when the number of cycle increases (each cycle being composed of a

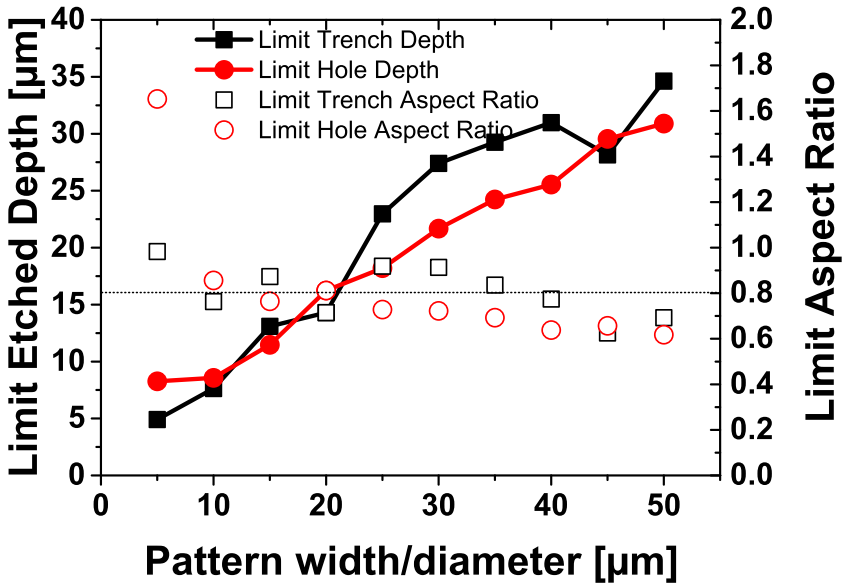

FIG. 2. (Color online) Limit etched depth and aspect ratio for which scalloping extends to $\sim 300 \mathrm{~nm}$. The measurements were taken on patterns etched with 90 cycles.

$7 \mathrm{~s}$-long passivation and a $13 \mathrm{~s}$-long etch step). As reported in Fig. 1(a), germanium is isotropically etched during the first etch step, leading to a round profile. During the second and subsequent cycles, the passivation efficiently protects the upper part of the profile, and the etched depth increases with the formation of scalloped sidewalls, as commonly observed during $\mathrm{Si}$ etching with time multiplexed etching. After few cycles (4-5 cycles in Fig. 1), the scallops from the subsequent cycles become hardly visible, and the sidewall becomes almost straight. Interestingly, the number of scallops visible on the top of the trench depends on the width/diameter of the pattern. For larger patterns, more scallops are observed. To put this in evidence, we plotted in Fig. 2 the limit pattern depth (including the mask) for which the scallops extends to $\sim 300 \mathrm{~nm}$, measured on patterns etched with 90 cycles. We also plot on this graph the limit aspect ratio corresponding to this limit pattern depth. It is clear that scallops are limited to the part of the patterns that has an aspect ratio below $\sim 0.8$. This phenomenon can be explained by a reduction in the scallop extent when the aspect ratio increases. Indeed, the etch rate decreases when the aspect ratio increases, which reduces the lateral and vertical etching per cycle. The 0.8 critical aspect ratio reported here corresponds to scalloping below $300 \mathrm{~nm}$. However, scalloping can still be observed for larger aspect ratio, but the extent is below $300 \mathrm{~nm}$.

These observations indicate that germanium can actually be etched using a time multiplexed plasma etch process developed for silicon and fast etching of deep patterns with a high aspect ratio (e.g., $3.7 \mu \mathrm{m} / \mathrm{min}$ average etch rate for trenches with a final aspect ratio of 11 in Fig. 1) can be obtained. In addition, the scallops are located in the upper part of the pattern corresponding to an aspect ratio of approximately 0.8 .

\section{B. Aspect ratio dependent etching}

One common limitation of plasma etching for deep etching of high aspect ratio holes or trenches is the reduction in the etch rate when the aspect ratio increases. Such a phenomenon is also observed during germanium etching, as reported in Fig. 3. Indeed, we can see that the etched depth does not 

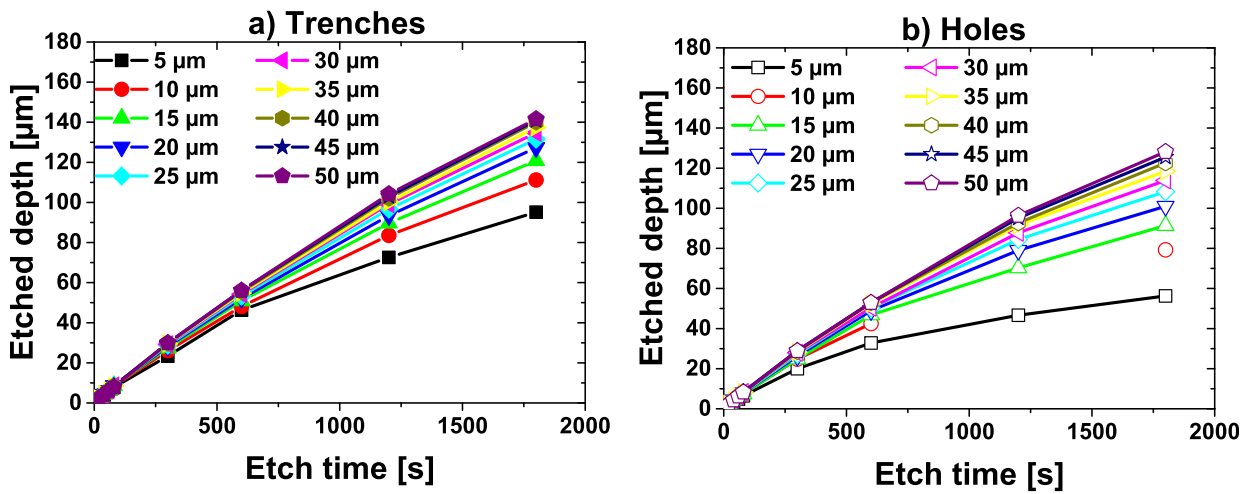

Fig. 3. (Color online) Etched depth as a function of the process time for patterns with $\mathrm{CD}$ of 5-50 $\mu \mathrm{m}$ : (a) trench patterns and (b) circular hole patterns.

linearly increase with the number of cycles or process time, and that the effect is more pronounced for smaller features, and for holes than for trenches. To evidence the aspect ratio dependence of the etch rate, we calculated the average instantaneous etch rate (ER) as a function of the average instantaneous aspect ratio (AR) using

$$
\begin{aligned}
& \operatorname{ER}\left(\mathrm{AR}_{i}\right)=\frac{d_{i}-d_{i-1}}{t_{i}-t_{i-1}}, \\
& \mathrm{AR}_{i}=\frac{d_{i}+t_{H M i}+d_{i-1}+t_{H M i-1}}{2 \mathrm{CD}},
\end{aligned}
$$

where $d_{i}$ and $t_{i}$ are the etched depth and time for the $i^{\text {th }}$ experiment and $t_{H M i}$ is the remaining hard mask thickness for the $i^{\text {th }}$ experiment (each experiment corresponding to a given number of cycles). $\mathrm{CD}$ is the critical dimension corresponding to the trench width or the hole diameter. As shown in Fig. 4, the average instantaneous etch rate only depends on the average instantaneous aspect ratio for a given pattern (trench or hole) and strongly decreases when the aspect ratio increases. This phenomenon is more important for holes than trenches. Based on Figs. 3 and 4, we can say that the dramatic decrease of the etch rate when the aspect ratio increases will make the etching of circular holes difficult with an aspect ratio larger than 10 in our process. However, no etch stop phenomenon has been reached yet since the instantaneous etch rate remains significant $(\sim 900 \mathrm{~nm} / \mathrm{min})$. For trench patterns, we can see that the etch rate remains larger than $2 \mu \mathrm{m} / \mathrm{min}$ even with an aspect ratio of
17, which will enable the etching of larger aspect ratio patterns. We can also notice that the aspect ratio of 0.8 corresponding to the limit to scalloping for trench and holes corresponds to a regime where the instantaneous etch rate has decreased by approximately $25 \%$ compared to the limit etching with an AR of 0 . This means that the instantaneous etch rate is approximately $25 \mathrm{~nm} / \mathrm{s}$ for an aspect ratio of 0.8 and therefore that the etched depth during 1 cycle $(13 \mathrm{~s})$ is around $325 \mathrm{~nm}$, consistent with the detection limit $(300 \mathrm{~nm})$ for the scallop.

To clarify the origin of these phenomena, we will analyze the specific effects of the passivation and the etch steps in the following.

\section{Mechanism of passivation step}

To determine the mechanism of the passivation step, we performed a 140 s-long deposition step on a previously patterned sample. The previously patterned sample is a germanium sample etched with 15 or 45 cycles that has been cleaned using an $\mathrm{O}_{2}$ plasma process followed by a $\mathrm{H}_{2} \mathrm{O}_{2}$ wet etch at room temperature. Such a sequence is believed to remove any fluorocarbon polymer and germanium oxide from the sample. As shown in Fig. 5, the long deposition step actually deposits a film inside the patterns. However, this film is observable only on surfaces in line of sight with the mask opening. In other words, no film is observable on any surface that cannot be reached by a plasma species with a straight and linear direction. In addition, the thickness of the deposit at the bottom of the patterns depends on the
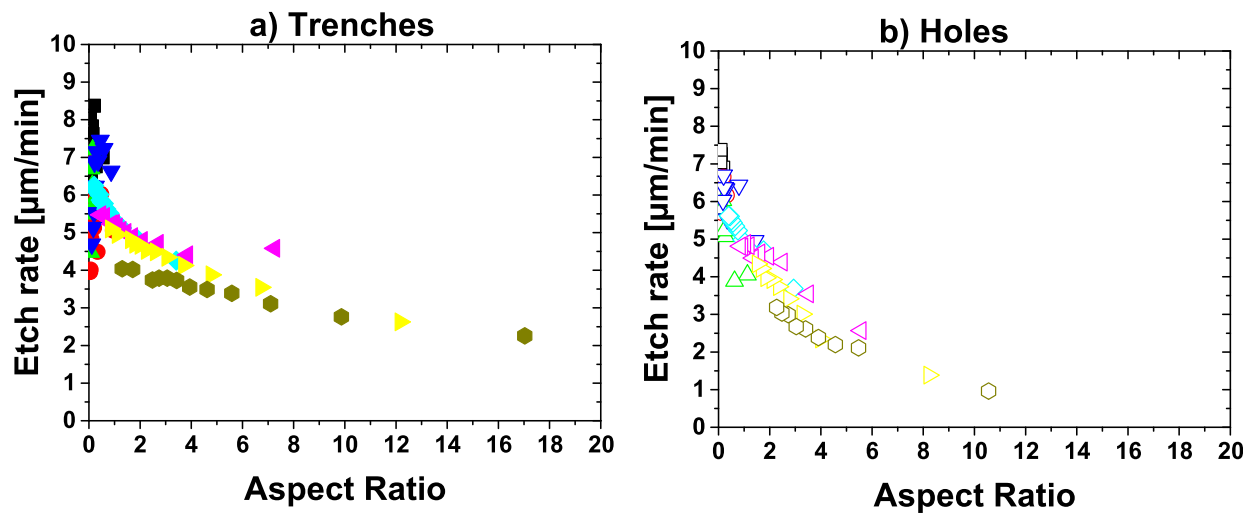

FIG. 4. (Color online) Average instantaneous etch rate as a function of the average instantaneous aspect ratio for patterns with CD of 5-50 $\mu$ m: (a) trench patterns and (b) circular hole pattern. The various symbols correspond to measurements for various numbers of cycles. 


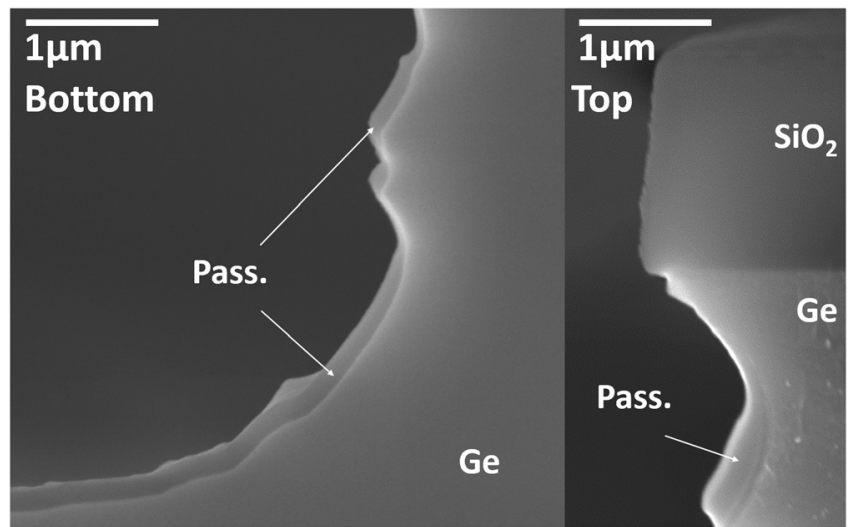

FIG. 5. SEM observation of fluorocarbon-based passivation layer deposited in previously patterned samples. Images are for a $100 \mu \mathrm{m}$-wide trench (left) and a $5 \mu \mathrm{m}$-wide trench (right).

pattern CD. We plotted in Fig. 6 the deposit thickness at the center of the bottom of the pattern as a function of the aspect ratio measured on SEM images. It is clear in Fig. 6 that the deposit thickness depends on the aspect ratio and decreases when the aspect ratio increases. Such a phenomenon is more pronounced for holes than for trenches.

The precursors for fluorocarbon species' deposition in a plasma are typically unsaturated fluorocarbon molecules. ${ }^{19,20}$ They can reach the surface either as neutral species or as ionized species. ${ }^{21,22}$ In addition, ion bombardment can favor the deposition of the fluorocarbon film by creating adsorption sites at the surface. Both the ion and neutral fluxes at the bottom of trenches and holes decrease when the aspect ratio increases-this effect being more pronounced for holes than for trenches. ${ }^{23}$ The reduction in the species flux at the bottom of the high aspect ratio trenches/holes can have several origins. (1) The shading effect consists of the reduction in the flux of species at the bottom of a feature because of the feature's wall that blocks the species. For isotropic species, the shading effect can be expressed by the reduction in the solid angle of collection because of the presence of the wall. For anisotropic species, the angular distribution function must also be taken into account. (2) For neutral species with a low sticking coefficient, the diffusion in holes and trenches can be assimilated to Knudsen diffusion since the mean free path is larger than the hole/trench CD. In this case, species travel from wall to wall without collisions and are assumed to be reemitted from the walls with a cosine law. This results in a reduction in the flux when the aspect ratio increases. ${ }^{24}$ (3) For charged species, the flux is reduced by the buildup of differential charges at the pattern sidewalls. Indeed, different angular distribution functions between negative (electrons) and positive (ions) species results in differential charges inside the pattern. The entrance potential depends then on the aspect ratio and reduces the ion flux and energy at the bottom of the patterns. ${ }^{25}$ In our case, the anisotropic behavior of the deposit thickness, and more particularly the nonobservable deposition on surfaces that are not in line of sight with the plasma, indicates that the deposit is either due to neutral species with

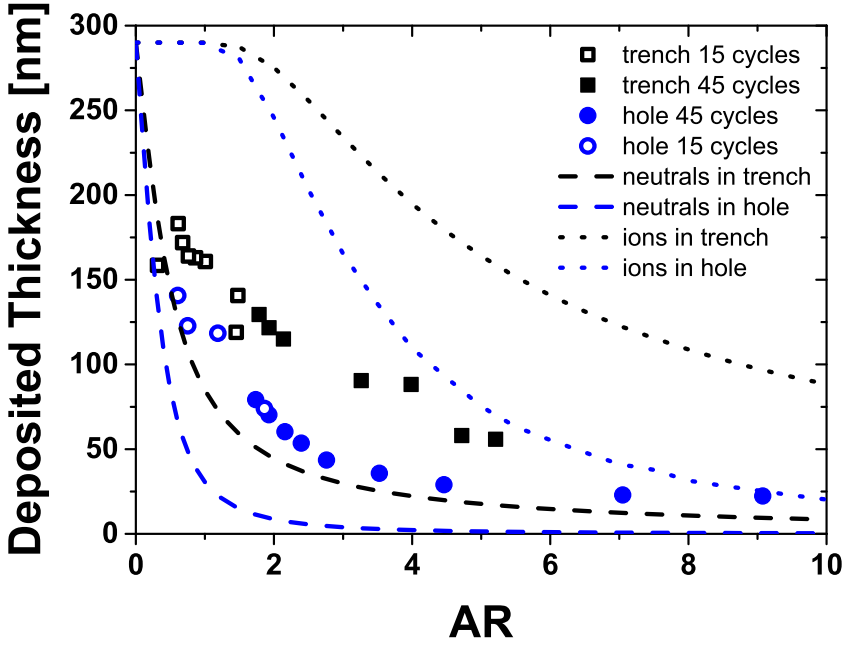

FIG. 6. (Color online) Thickness of the fluorocarbon-based passivation layer deposited at the center of the bottom of the patterns, and evolution of the modeled deposited thickness for neutral-based deposition with a sticking coefficient of 1 and for ion-based deposition for ions transversal and longitudinal energies of 1 and $15 \mathrm{eV}$, respectively. Thickness is measured on SEM pictures.

a sticking coefficient close to 1 or to ion species. Indeed, neutral species with a sticking coefficient significantly below 1 would bounce around before sticking onto the sidewalls and would therefore lead to a significant deposit on surfaces that are not in line of sight with the plasma. Therefore, we can rule out Knudsen diffusion as a dominant mechanism for passivation precursors' transport in the holes.

Assuming the deposition by ions, we can estimate the deposited thickness at the center of the pattern using

$$
d \propto \int_{\varphi=0}^{2 \pi} \int_{\vartheta=0}^{\alpha} \operatorname{IADF}(\vartheta) \sin (\vartheta) d \vartheta d \varphi,
$$

where $\vartheta$ and $\varphi$ are the latitude and azimuth angle in polar coordinate, taking a central point at the bottom of the hole/ trench as the origin, respectively, and $\operatorname{IADF}(\vartheta)$ is the ion angular distribution function. Assuming a Maxwellian velocity distribution for the transversal energy $E_{T}$ and a given longitudinal energy $E_{L}$, the ion angular distribution function (IADF) can be expressed by (see Appendix)

$$
\operatorname{IADF}(\vartheta) \propto \frac{e^{\left(-\frac{E_{L} \tan ^{2} \vartheta}{E_{T}}\right)}}{\cos ^{2} \vartheta} .
$$

The angle $\alpha$ is the critical angle above which a straight line starting from the origin would strike a wall of the pattern. This angle is defined by $\alpha=\arctan (1 / 2 \mathrm{AR})$ for a circular hole and $\alpha=\arctan (1 / 2 \mathrm{AR} \sin \varphi)$ for an infinite trench, where AR is the aspect ratio.

For a transverse ion energy of $15 \mathrm{eV}$ and a longitudinal (thermal) energy of $1 \mathrm{eV}$, we plotted in Fig. 6 the ion deposited thickness at the center of holes and trenches, normalized to obtain a deposited thickness of $290 \mathrm{~nm}$ for an aspect ratio of 0 , measured by exposing a blank germanium sample to the $140 \mathrm{~s}$-long passivation step. In this case, it is clear that the evolution of the actual deposited thickness follows a 
different evolution to the one of the ion flux as a function of the AR. The former steeply decreases for small aspect ratio while the latter having a plateau for aspect ratios below 2 and decreasing afterward. Note that the shape would be similar assuming different ion longitudinal and transversal temperatures, except for different limit aspect ratios before the drop of the deposition rate. In addition, the deposited thickness assuming deposition by ions only is overestimated for all aspect ratios. Finally, by looking closely at Fig. 5, we can see significant deposition under the mask that would require a significant flux of ions entering the pattern with an angle of $50^{\circ}$ with respect to the normal, which is not realistic with our plasma conditions. Therefore, we can conclude that ioninduced deposition is not the dominant phenomenon for the passivation layer deposition.

On the other hand, assuming the deposition by neutral species with a sticking coefficient of 1 , the deposit thickness would be proportional to the solid angle of collection $\Omega$. The proportionality constant can be determined using the deposited thickness on the blank germanium $(290 \mathrm{~nm})$. We plotted in Fig. 6 the calculated deposited thickness at the bottom of the patterns. It is clear that the actual deposited thickness at the bottom of the holes and trenches presents a similar evolution but exceeds the calculated value. This indicates that deposition in line of sight by neutrals with a sticking coefficient of 1 is not the solely responsible for passivation layer deposition. The underestimation of the deposition could be explained by a sticking coefficient slightly below 1 that would help driving the neutral species toward the bottom of the patterns and by the contribution of ionic species. However, the similar evolution indicates that neutrals are the dominant species for passivation layers' deposition. There are several contradictory reports on the deposition mechanism for fluorocarbon films by fluorocarbon plasmas in the literature. For example, Saraf et al. ${ }^{22}$ conclude that fluorocarbons are deposited by ions, while Takahashi et al. ${ }^{26}$ conclude that CF radicals are responsible for polymer deposition. Oehrlein et al. proposed a deposition by neutrals enhanced by the ion bombardment. ${ }^{27}$ Booth mentioned that neutral and charged heavy $\mathrm{C}_{\mathrm{x}} \mathrm{F}_{\mathrm{y}}$ species are responsible for the deposition. ${ }^{28}$ Most probably, ions and neutrals participate in species deposition, one or the other being dominant depending on the plasma conditions. In our plasma conditions, neutrals' deposition seems to dominate.

Therefore, we can attribute the deposition of the passivation layer mostly to neutral species that have a large sticking coefficient. During the actual process, the sputtering of the passivation layer at the bottom of the patterns at the beginning of the etch step can also participate in depositing fluorocarbon species along the height of the patterns, but this effect cannot be captured with the experiments presented in this part. ${ }^{29,30}$

\section{Mechanism of etching step}

To determine the mechanisms of the germanium etching during the etching step, we used a similar methodology to the one used for the passivation investigation. Previously patterned germanium samples have been exposed to a 52 s-long etch process (corresponding to four times the usual step time). The samples were then observed by SEM, as illustrated in Fig. 7. We can see that after the etching process, the upper part of the pattern is blown out while the bottom part is hardly changed. In order to precise the evolution of the profile, we extracted lateral etched thickness by comparing the $\mathrm{CD}$ along the pattern height with a step size of $5 \mu \mathrm{m}$ for the pattern with and without the extended etch step. To compensate for SEM errors due to misalignment and nonperfect process reproducibility, the data were shifted so that the asymptotic value at large AR equals 0 . The plotted values in Fig. 8 correspond to two times the lateral etching during the 52 s-long etch step. It is clear that the increase in pattern dimension is only localized in the upper part of the trench that corresponds to an aspect ratio below $\sim 2$. This signature is representative of chemical etching from neutral species with reaction probability close to 1. Indeed, the solid angle of collection at the edge of a trench having an aspect ratio of 2 is reduced by more than $85 \%$ compared to a flat surface. Reactive fluorine radicals originating from the plasma stick on the sidewalls and react with germanium to form volatile etch by products. Therefore, the flux of free fluorine at depth corresponding to an aspect ratio larger than 2 is reduced by a factor of $85 \%$ for trenches. This is a similar phenomenon to the passivation with species having a sticking coefficient of 1 . We can also see in Fig. 8 that the etched depth hardly increases during the extended etch step. However, the difference in etched depth is too small to be precisely quantified and is within the incertitude of the SEM measurements and process reproducibility. Therefore, we cannot conclude on the impact of the etch step on the etched depth, but the low etch rate at the bottom tends to indicate that the etching is mostly due to radicals and that ions play a minor role in the etching of germanium in $\mathrm{SF}_{6} / \mathrm{O}_{2}$ plasma.

In these conditions, one would expect no significant etching for aspect ratio larger than $\sim 6$ and $\sim 1.5$ for trench and hole patterns, respectively. These aspect ratios correspond to $95 \%$ of reduction in the solid angle of collection. Even if a strong aspect ratio dependent etching was observed in part $\mathrm{B}$, we could observe significant etching even for holes with an aspect ratio larger than 6 . To explain this discrepancy, we performed an additional experiment by exposing a previously patterned sample to an extended etch step. However, the previously patterned sample was not cleaned using the $\mathrm{O}_{2}$ plasma and the $\mathrm{H}_{2} \mathrm{O}_{2}$ wet etch, meaning that passivation layers remained at the patterns' sidewalls before the extended etch step. As shown in Figs. 7(c) and 7(f), the evolution of the profile is drastically different when the passivation layers were present at the pattern sidewalls. In this case, the blowout is only localized at the bottom of the pattern, and a significant increase in etched depth is observed. This shows that fluorocarbon species present at the sidewalls of the patterns efficiently passivate the sidewalls and prevent the lateral etching. More importantly, it reduces the sticking coefficient and reaction probability of fluorine on the sidewalls, which enables the Knudsen-like diffusion of fluorine radicals down to the bottom of the patterns. The reaction of the radicals with germanium at the bottom of the trench is possible, thanks to the sputtering of the passivation layer by 


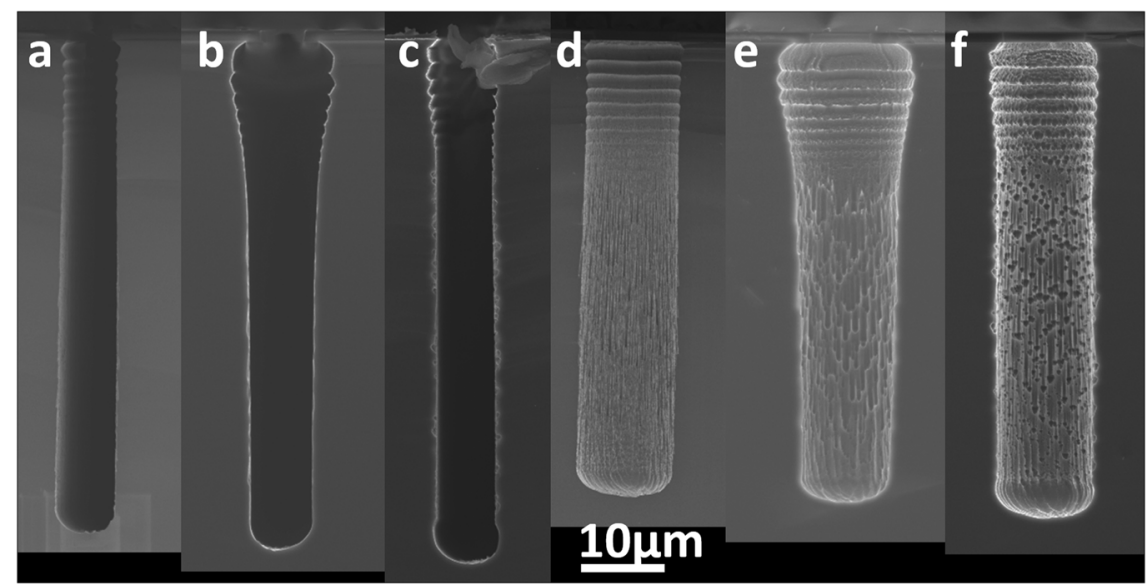

FIG. 7. Germanium patterns etched using time multiplexed plasma: (a) $5 \mu$ m-wide trench etched with 45 cycles; (b) $5 \mu$ m-wide trench etched with 45 cycles followed by $\mathrm{O}_{2}$ plasma and $\mathrm{H}_{2} \mathrm{O}_{2}$ cleaning and an extended (52 s) etch step; (c) $5 \mu$ m-wide trench etched with 45 cycles followed by an extended (52 s) etch step; (d) $10 \mu$ m-diameter circular hole etched with 45 cycles; (e) $10 \mu$ m-diameter circular hole etched with 45 cycles followed by $\mathrm{O}_{2}$ plasma and $\mathrm{H}_{2} \mathrm{O}_{2}$ cleaning and an extended (52 s) etch step; and (f) $10 \mu \mathrm{m}$-diameter circular hole etched with 45 cycles followed by an extended (52 s) etch step.

ion bombardment. Note that the sidewall passivation is also partially etched during the etch step, as indicated by "mouse bite" tiny holes along the height of the patterns, especially visible in Fig. 7(f). Therefore, there is a competition between sidewall passivation etching and Knudsen diffusion of fluorine down to the bottom of the patterns.

\section{DISCUSSION AND CONCLUSION}

We have demonstrated that germanium can be etched using a time multiplexed process comparable to the process developed for silicon deep etching. As silicon etching, germanium etching presents a strong aspect ratio dependent etching, and the pattern sidewalls present scalloping. The scalloping is located at the upperpart of the trench and is more significant within the part with a depth to $\mathrm{CD}$ ratio below 0.8 . We have shown that the passivation is mostly

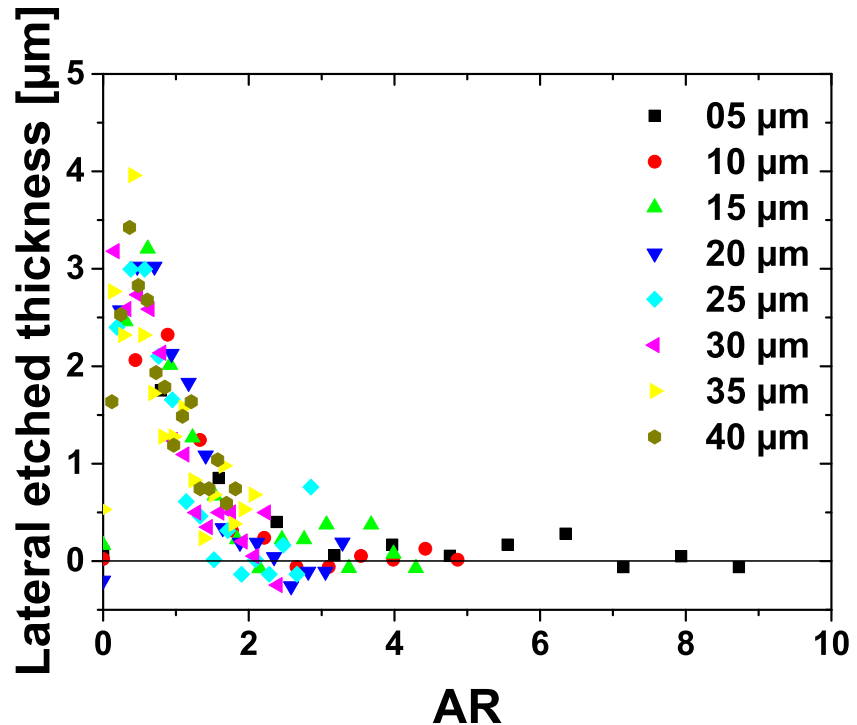

FIG. 8. (Color online) Lateral increase of trench patterns width after an extended (52 s) etch step. The lateral increase is measured along the height of trenches and plotted as a function of the aspect ratio at the measurement location. attributed to neutrals with a sticking coefficient close to 1 . Therefore, most of the deposition occurs on the top part of the sidewalls, and the passivation layer thickness at the bottom of the pattern decreases when the aspect ratio increases. During the etching step, fluorine radicals can react with germanium with a reaction probability close to 1 , leading to lateral etching on the upper part of the trench when the sidewalls are not passivated. When passivation remains on the sidewalls, the fluorine sticking coefficient is reduced, leading to Knudsen-like diffusion of fluorine down to the bottom and germanium etching at the bottom. The passivation layer is also partially etched by fluorine. The ions do not directly participate in the etching, except by clearing the passivation layer from the etch front.

The difference between the passivation (neutrals diffusion with a sticking coefficient close to 1) and the etching (Knudsen-like diffusion when the sidewalls are passivated) mechanisms should lead to a deficit of passivation layers for aspect ratios larger than $\sim 6$ and 1.5 for trenches and holes, respectively, compared to the flux of fluorine, and therefore to an enlargement of the pattern when the process proceeds. This phenomenon is not observed here, which indicates that passivation layers are present along all the depth of the pattern. Such passivation layers were not observed in the experiments of part $\mathrm{C}$, but can be attributed to the sputtering of the fluorocarbon species from the etch front during the etch step that leads to passivation layer deposition on the sidewalls close to the etch front. ${ }^{29,30}$

Therefore, we can say that complex passivation and etch mechanisms are involved in the etching of high aspect ratio patterns in germanium using a time multiplexed plasma etching process. Such processes are suitable to deep etch germanium with a large etch rate. Etching circular hole patterns with an aspect ratio larger than 10 will be difficult because of the reduced flux of fluorine at the bottom of the patterns in Knudsen-like diffusion regime. For trench patterns, the aspect ratio larger than 15 can be obtained with a process developed for silicon deep etching. 


\section{ACKNOWLEDGMENTS}

LN2 is a joint International Research Laboratory (Unité Mixte de Recherche UMI 3463) funded and co-operated by CNRS (France) and Université de Sherbrooke (Canada) as well as INSA Lyon, ECL, CPE, Université Joseph Fourier (UJF), as well as the French national nanofabrication network RENATECH. The authors also acknowledge MarieJosée Gour for fruitful discussions. The financial support from NSERC is acknowledged.

\section{APPENDIX: CALCULATION OF THE ION ANGULAR DISTRIBUTION FUNCTION}

The relationship between the ion angle $\vartheta$ with respect to the surface normal and the transversal $\left(v_{T}\right)$ and longitudinal $\left(v_{L}\right)$ ion energy is

$$
\vartheta=\operatorname{Arctan} \frac{v_{T}}{v_{L}} .
$$

Therefore

$$
d v_{T}=\frac{v_{L}}{\cos ^{2} \vartheta} d \vartheta .
$$

Assuming a Maxwell-Boltzmann distribution function for the ions in the plasma, the ion velocity distribution function (IVDF) is expressed by

$$
\operatorname{IVDF}\left(v_{T}\right)=\left(\frac{m}{2 \pi k T_{T}}\right)^{\frac{1}{2}} e^{\left(-\frac{m T^{2}}{2 k T_{T}}\right)} .
$$

With $T_{T}$ the ion temperature in the plasma, $m$ the ion mass and $n$ the plasma density.

The ion flux can be calculated by integrating the IVDF or the IADF

$$
\int_{0}^{\infty} \operatorname{IVDF}(v) d v=\int_{\vartheta=0}^{\frac{\pi}{2}} \operatorname{IADF}(\vartheta) d \vartheta
$$

By changing the variable in the IVDF integration, we can express

$$
\begin{aligned}
\int_{0}^{\infty} \operatorname{IVDF}(v) d v & =\int_{v_{T}=0}^{\infty} n_{x}\left(\frac{m}{2 \pi k T_{T}}\right)^{\frac{1}{2}} e^{\left(-\frac{m v_{T}^{2}}{2 k T_{T}}\right)} d v_{T} \\
& =\int_{\theta=0}^{\frac{\pi}{2}} n_{x}\left(\frac{m}{2 \pi k T_{T}}\right)^{\frac{1}{2}} e^{\left(-\frac{m v_{L}^{2} \tan ^{2} \theta}{2 k T_{T}}\right)} \frac{v_{L}}{\cos ^{2} \theta} d \theta .
\end{aligned}
$$

Therefore, the IADF can be expressed by

$$
\operatorname{IADF}(\vartheta)=n\left(\frac{m}{2 \pi k T_{T}}\right)^{\frac{1}{2}} e^{\left(-\frac{m v_{L}^{2} \tan ^{2} \theta}{2 k T_{T}}\right)} \frac{v_{L}}{\cos ^{2} \theta} .
$$

The ion velocity can be replaced by the temperature using: $k T_{i}=1 / 2\left(m v_{i}^{2}\right)$.

Therefore

$$
\operatorname{IADF}(\vartheta)=n \sqrt{\frac{T_{L}}{\pi T_{T}}} \frac{e^{\left(-\frac{E_{L} \tan ^{2} \vartheta}{E_{T}}\right)}}{\cos ^{2} \vartheta} \propto \frac{e^{\left(-\frac{E_{L} \tan ^{2} \vartheta}{E_{T}}\right)}}{\cos ^{2} \vartheta} .
$$

${ }^{1}$ J. R. Heath and F. K. LeGoues, Chem. Phys. Lett. 208, 263 (1993).

${ }^{2}$ C. Fang, H. Föll, and J. Carstensen, Nano Lett. 6, 1578 (2006).

${ }^{3}$ M. Lindblom, J. Reinspach, O. von Hofsten, M. Bertilson, H. M. Hertz, and A. Holmberg, J. Vac. Sci. Technol. B 27, L1 (2009).

${ }^{4}$ G. S. Oehrlein, T. D. Bestwick, P. L. Jones, M. A. Jaso, and J. L. Lindström, J. Electrochem. Soc. 138, 1443 (1991).

${ }^{5}$ A. Campo, C. Cardinaud, and G. Turban, J. Vac. Sci. Technol. B 13, 235 (1995).

${ }^{6}$ A. Campo, C. Cardinaud, and G. Turban, Plasma Sources Sci. Technol. 4, 398 (1995).

${ }^{7}$ G. F. McLane, M. Dubey, M. C. Wood, and K. E. Lynch, J. Vac. Sci. Technol. B 15, 990 (1997).

${ }^{8}$ C. Monget, A. Schiltz, O. Joubert, L. Vallier, M. Guillermet, and B. Tormen, J. Vac. Sci. Technol. B 16, 1833 (1998).

${ }^{9}$ J. Y. Choe, I. P. Herman, and V. M. Donnelly, J. Vac. Sci. Technol. A 16, 3266 (1998)

${ }^{10}$ K.-H. Shim, Y.-H. Kil, H.-D. Yang, B.-K. Park, J.-H. Yang, S. Kang, T. S. Jeong, and T.-S. Kim, Mater. Sci. Semicond. Process. 15, 364 (2012).

${ }^{11}$ K.-H. Shim, H.-Y. Yang, Y.-H. Kil, H.-D. Yang, J.-H. Yang, W.-K. Hong, S. Kang, T. S. Jeong, and T.-S. Kim, Electron. Mater. Lett. 8, 423 (2012).

${ }^{12}$ C. Wongwanitwattana, V. Shah, M. Myronov, E. H. C. Parker, T. Whall, and D. R. Leadley, J. Vac. Sci. Technol. A 32, 031302 (2014).

${ }^{13}$ M. C. Peignon, C. Cardinaud, G. Turban, C. Charles, and R. W. Boswell, J. Vac. Sci. Technol. A 14,156 (1996).

${ }^{14}$ H. V. Jansen, M. J. de Boer, S. Unnikrishnan, M. C. Louwerse, and M. C. Elwenspoek, J. Micromech. Microeng. 19, 033001 (2009).

${ }^{15}$ F. Laermer and A. Schlip, U.S. patent 5,501,893 (27 November 1993).

${ }^{16}$ K. R. Williams, K. Gupta, and M. Wasilik, J. Microelectromech. Syst. 12,761 (2003)

${ }^{17}$ S. Schicho, A. Jaouad, C. Sellmer, D. Morris, V. Aimez, and R. Arès, Mater. Lett. 94, 86 (2013).

${ }^{18}$ B. Lee Sang, M. J. Gour, A. Jaouad, S. Ecoffey, M. Darnon, B. Sadani, A. Souifi, and D. Drouin, Microelectron. Eng. 141, 68 (2015).

${ }^{19}$ C. B. Labelle, V. M. Donnelly, G. R. Bogart, R. L. Opila, and A. Kornblit, J. Vac. Sci. Technol. A 22, 2500 (2004).

${ }^{20}$ W. W. Stoffels, E. Stoffels, and K. Tachibana, J. Vac. Sci. Technol. A 16, 87 (1998).

${ }^{21}$ M. J. Sowa, M. E. Littau, V. Pohray, and J. L. Cecchi, J. Vac. Sci. Technol. A 18, 2122 (2000).

${ }^{22}$ I. R. Saraf, M. J. Goeckner, B. E. Goodlin, K. H. R. Kirmse, C. T. Nelson, and L. J. Overzet, J. Vac. Sci. Technol. B 31, 011208 (2013).

${ }^{23}$ R. A. Gottscho, C. W. Jurgensen, and D. J. Vitkavage, J. Vac. Sci. Technol. B 10, 2133 (1992).

${ }^{24}$ J. W. Coburn and H. F. Winters, Appl. Phys. Lett. 55, 2730 (1989).

${ }^{25}$ G. S. Hwang and K. P. Giapis, Appl. Phys. Lett. 71, 458 (1997).

${ }^{26}$ K. Takahashi, M. Hori, and T. Goto, J. Vac. Sci. Technol. 14, 2011 (1996).

${ }^{27}$ G. S. Oehrlein, Y. Zhang., D. Vender, and M. Haverlag, J. Vac. Sci. Technol. A 12, 333 (1994).

${ }^{28}$ J. P. Booth, Plasma Sources Sci. Technol. 8,249 (1999).

${ }^{29}$ H. Huübner, J. Electrochem. Soc. 139, 3302 (1992).

${ }^{30}$ O. Luere, E. Pargon, L. Vallier, B. Pelissier, and O. Joubert, J. Vac. Sci. Technol. B 29, 011028 (2011). 